\title{
Modification of Rectal Myofascial Repair in Abdominoplasty for Patients with Widely Divaricated Recti
}

\author{
MEDHAT S. HASSAN, M.D. \\ The Department of Plastic, Reconstructive \& Burn Surgery, Faculty of Medicine, Menoufia University, Egypt
}

\begin{abstract}
Background: To gain the best aesthetic outcome after abdominoplasty for patients with weak abdominal wall muscles has been the ultimate goal for plastic surgeons and patients. Myofascial repair is one of the modern techniques to have a flat abdomen and a perfect waist with accepted rehabilitative outcome. But as any technique, it has its benefits and drawbacks.

Aim of the Study: This study aims to document and evaluate the durability of modified myofascial repair as a method for musculoapneurotic reconstruction in abdominoplasty for patients with moderate to severe weak lax abdomen.
\end{abstract}

Patients and Methods: Thirteen patients were included in this study for the purpose of performing abdominoplasty to treat weak lax abdomen over the period of 2 years from January 2017 to February 2019. Follow-up period for each case was at least 6 months for the functional outcome and patient satisfaction.

Results: Changes in spirometry values before and after operation were recorded and tolerated by all patients. Waist measures taken before and after operation showed great improvement. Patients satisfaction was extremely high and there were no evidences for secondary hernias.

Conclusion: Myofacial repair modification is a durable method to gain a good aesthetic and functional output in abdominoplasty for patients with severe abdominal laxity.

Key Words: Divaricated recti - Rectal myofascial repair Abdominoplasty.

\section{INTRODUCTION}

Abdominoplasty objectives have been changing over decades, related directly to the technological breakthrough that happened in the last few years which raised the standard lifestyle demands and expectations. Traditional abdominoplasty objectives for sever deformities included the following: (1) Removal of excess fat and skin, (2) Correction of muscle diathesis, and (3) Transposition of the umbilicus to its new location on the anterior abdominal wall. But with time, these objectives changed to the following: (1) Place the incision within the bikini line, (2) Flatten and tighten the abdomen, (3) Decrease the size of the waist line, (4) Decrease fat in the abdomen, flanks and the iliac area, (5) Change the shape of pubis to a more youthful form, (6) Create a well formed xiphoumbilical depression [1].

With the introduction of other techniques to fulfill these objectives such as suction assisted lipectomy [2], it was still not enough for correction of the deformities in the waist area, as the problem lies in the significant divarication in the recti muscles rather than the fat content [3].

The plication of the musculoapneurotic layer was first performed by non-absorbable sutures for recti at 1967 [4]. After that many modifications were introduced such as using braided sutures in combination with non-absorbable sutures, in addition to multiple layered repairs [5-7]. However, with all these modifications still recurrence following plication was reported. It was reported that $40 \%$ of 63 women who performed abdominoplasty showed recurrence on ultrasound evaluation [8].

Also concerns were expressed that rectus plication could actually increase the risk of hernia development. This led some surgeons to believe that not repairing rectus diastasis is better in case of presence of large intra-abdominal fat volume would be a wise decision for fear of recurrence and ventral hernia development [9]. Placing running and interrupted sutures under tension can cut through the anterior rectus fascia like knife in cheese, which is considered the main reason of non-durability of rectal approximation by sutures due to the stretching rectal diastasis effect [10].

The main purpose for introduction of myofascial repair was reconstruction of congenital rectus abdominis diastasis where diastasis was considered 
midline abdominal defect. That was when DiBello in 1996 introduced sliding myofascial release and repair as an alternative technique for the closure of recurrent ventral hernias. It relied on creating facial raw area to promote more solid healing between both recti [11].

Based on these concepts and facts, Rameriz in 2000 [1] introduced myofascial release and repair as a main step in abdominoplasty. The main purpose is to allow centrifugal forces of the rectus muscles to push the muscles toward the midline facilitating closure with decreased tension, also it allows better pull of external oblique muscles for redefining and decreasing the waistline measurements.

In patient with severe rectus diastasis, performing myofascial release and repair would be quiet difficult due to the wide distance between both recti, this will cause increased tension along the line of repair due to the relevant increased intraabdominal pressure after repair. As a result, the patient may complain from postoperative pain and difficulty in breathing.

To overcome these problems, this study was performed to introduce a modification in myofascial release and repair, which will help to raise the curve of satisfaction of patients by giving better results with fewer problems with abdominoplasty.

\section{PATIENTS AND METHODS}

This is a prospective study which was performed from January 2017 till February 2019 at Plastic Surgery Department, Menoufia University Hospital. The study included patients undergoing abdominoplasty with moderate to severe abdominal laxity. Patients with chronic respiratory disorders, smoking, taking regular corticosteroids or anticonvulsant drugs or with systemic connective tissue disorders were excluded from this study.

\section{Pre-operative management:}

At first, detailed history taking with general and local examination of the patient was done to record any risk factors such as diabetes mellitus, hypertension and repeated pregnancy. Routine laboratory investigations including complete blood count, kidney and liver enzymes, random blood sugar, prothrombin time \& ratio and hepatitis B, $\mathrm{C}$ markers and also chest X-ray was also performed to complete evaluation of the general condition of the patient. A detailed consent concerning operative procedure was taken, in addition to taking a standard photo for the trunk pre-operatively.
Spirometry was performed pre-operatively to measure the forced vital capacity, forced expiratory volume in one second and results were assessed according to the American Thoracic Society criteria.

Proper bowel preparation for patients was necessary to decrease bowel distention after operation, which would decrease intra-abdominal pressure and relatively decrease risk of developing deep venous thrombosis, preparation including clear liquid diet for 2 to 3 days before surgery, enemas, laxatives and antibiotics decreasing intestinal flora producing gas.

At last, measurements of the waist circumference at the midpoint between the last rib and the iliac crest were recorded for each patient preoperatively.

\section{Pre-operative markings:}

Markings were made in the standing position. Reference lines were determined including groin lines with direct extension into the upper iliac areas, central midline of the abdomen, parallel lines to the central midline ( 6 to $9 \mathrm{~cm}$ ) that determine the outer border of the rectus abdominis muscle. Another horizontal line perpendicular to the midline is made across the abdomen usually at the level of the navel. These reference lines are useful for markings of the planned flap excision as symmetrical as possible.

A lazy $U$ shaped incision line was marked after lifting the pubic skin approximately $5-7 \mathrm{~cm}$ above the anterior vulvar commissure and extending to the groin areas toward the anterior superior iliac spine on both sides.

Then a lazy $\mathrm{M}$ incision line was marked with the central point above the umbilicus and the highest points on both sides of the central point were at the level of linea semilunaris. Then the line goes down gradually to the uppermost ends of the lower incision line mark.

\section{Operation:}

Surgery was performed under general anesthesia with the patient in the supine position. Crepe bandage was applied to lower extremities and the patients inserted a Foley catheter. Dissection is preceded by liposuction for the flanks and epigastric areas only after injection of a reasonable amount of tumescent fluid. Liposuction performed at the epigastric area above the position of the new umbilicus is suctioned up to the sub dermal layer to create xipho-umbilical depression. 
First step of dermolipectomy follows, in which incision of the skin along the $\mathrm{U}$ shaped marked line, was performed, flap elevation with electro cautery was done, large perforators were handled by sutures to prevent post-operative bleeding and dissection of the flap was extended up to the costal margins and the xiphoid process after completing incision of the marked lines.

\section{Myofascial release and repair:}

The usual incision in the traditional technique is done along the anterior rectus sheath at the junction of the inner one third and the middle third of the rectus sheath width, but in our study and because of the severe abdominal laxity, the incisions were made at half of the rectus width. These incisions begin from the xiphoid process superiorly to the pubis inferiorly marked first before incision by electro cautery.

The myofascial repair aims to push the rectus muscles toward the midline facilitating closure with decreased tension; it also allows better pull of the external oblique muscles, which will have a significant effect in decreasing the waist measurements.

This was traditionally achieved through a three layered closure procedure in which: The two inner slips of the anterior sheath are closed using continuous plus interrupted propylene 0 sutures as the first layer, both recti are sutured to each other by interrupted vicryl $2 / 0$ as the second layer Fig. (1) and the two outer slips of the anterior rectus sheath are closed using continuous plus interrupted propylene 0 sutures as the third layer Fig. (2).

BUT in patients with severe abdominal laxity, the previous procedure was not enough to approximate both recti towards the midline without increased tension on the sutures of the closed layers, especially the third layer. That is why it would be mandatory to apply another step to solve this problem.

In this study, small multiple marks were added along the linea alba in the midline, each mark was about $3-4 \mathrm{~cm}$ in length with a distance of $5-6 \mathrm{~cm}$ separating between them Fig. (3). Following that, Elliptical incisions were performed along these marks with the width of approximately $0.75 \mathrm{~cm}$ on both sides of the midline. Excision of these ellipses was done and the defects were closed by interrupted propylene 0 sutures. Other interrupted propylene 0 sutures were applied in the distances between the performed defects with $1 \mathrm{~cm}$ intervals to allow better approximation and decrease tension on the sutures closing the defects. After finishing, we perform the three layered myofascial repair steps, to end by having four layered myofascial repair Fig. (4). The changes in the intraoperative airway pressure were recorded before and after myofacial repair for further evaluation.

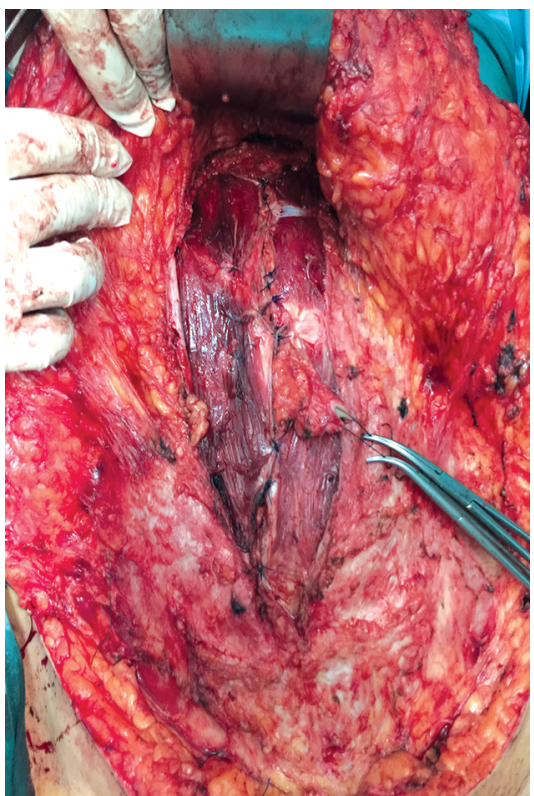

Fig. (1): Repair of the $1^{\text {st }}, 2^{\text {nd }}$ and $3^{\text {rd }}$ layers of the four layered myofascial repair.

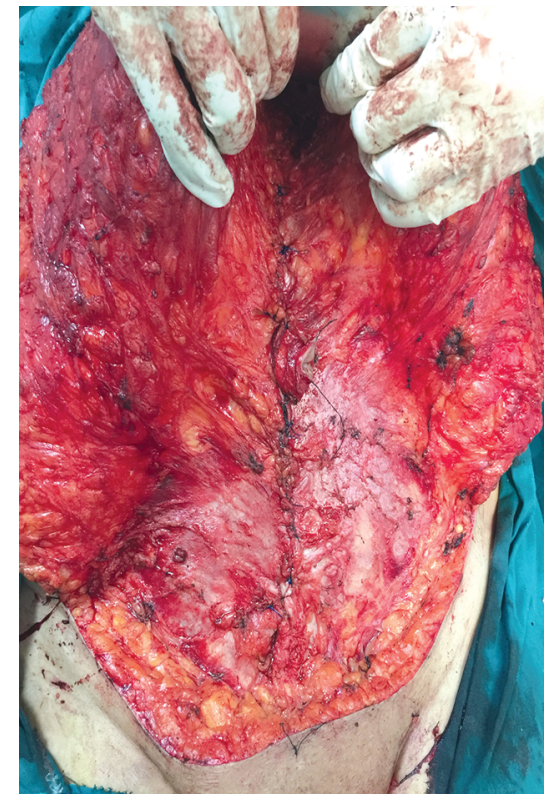

Fig. (2): Closure of the outer most layer in myofascial repair.

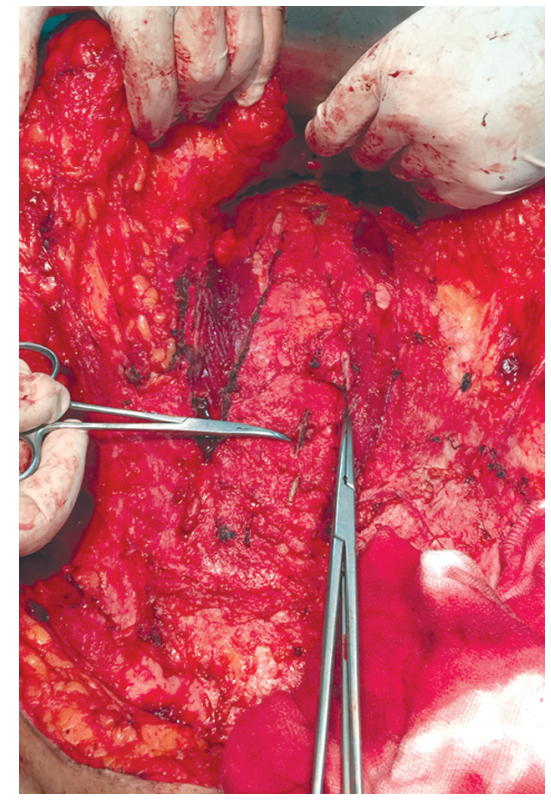

Fig. (3): Sites of the elliptical incisions in the linea alba at the beginning of the four layered myofascial repair. 


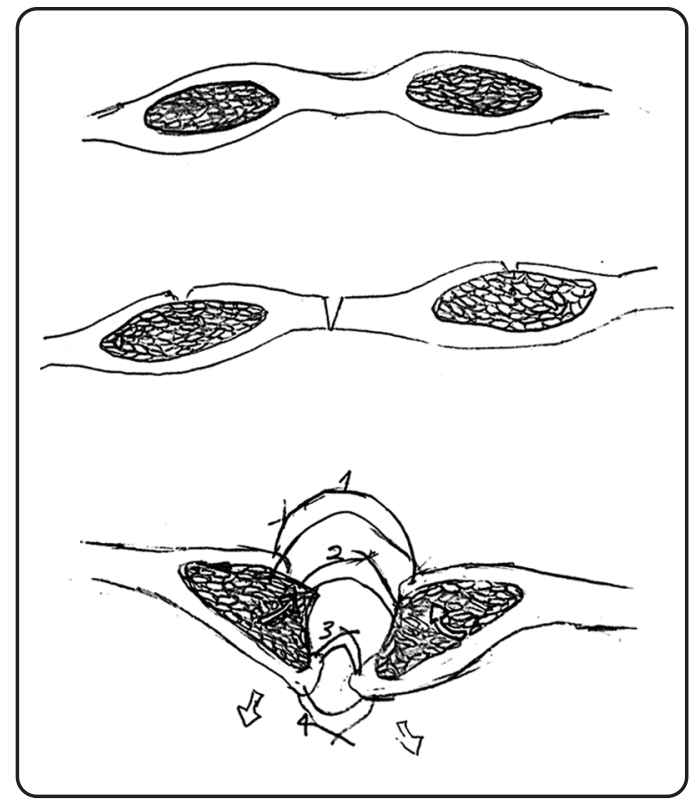

Fig. (4): Planes of dissection and repair in a four layered myofascial repair: (1) Outer most layer of the rectus sheath, (2) Both recti muscles, (3) Inner layer of the rectus sheath, (4) Sutures at the elliptical incisions of the linea alba and in between.

After myofascial repair, operative table was bent to 45 degrees to perform second step of dermolipectomy in which: The marked $M$ shaped incision line was matched against the lower incision and adjusted before flap trimming, Umbilicoplasty was done next, removal of excess skin and fat along the $\mathrm{M}$ shaped incision line in a perpendicular manner followed umbilicoplasty, and closure of the abdominal flap in a three layered manner was performed at the end. Suction drains were applied under the abdominal flap and patients were put in an appropriate abdominal binder.

\section{Post-operative management:}

After operation, intravenous fluids and antibiotics were given to the patients for 24 hours followed by oral fluids and soft diet. Ambulation was started from the first day. Drains were removed after 4 to 6 days. And for several weeks, the patients were advised to continue using the abdominal binder in addition to light exercise.

Follow-up of the patients was performed monthly for a duration of 6 months, it included: Repeating spirometry to measure the new forced vital capacity and forced expiratory volume in one second, taking photographs for the abdomen and flanks and measuring the new waist circumference. All data collected was put in a comparison to operative data and evaluated to monitor the progress of improvement.

\section{RESULTS}

This study included thirteen (13) patients, all with severe abdominal laxity showing divaricated recti by examination. Their age ranged from 34 to 56 years with the mean value of 47.2 years, their weight ranged from 74 to $115 \mathrm{~kg}$ with the mean value of 97.5 years, their height ranged from 153 to $168 \mathrm{~cm}$ and their body mass index ranged 33 to 35.5 kilograms per square meter.

About 8 patients were performed under general anesthesia and 5 patients were under spinal anesthesia. All patients had the four layered myofascial repair with application of this modification.

All patients showed mild increase in the intraoperative airway pressure values before and after myofascial repair, but oxygen saturation of each patient was normal and did not change all time of the operation.

Changes in the spirometry values were recorded and evaluated carefully. As all patients with respiratory disorders were excluded from this study, each patient pre-operatively showed normal Forced Vital Capacity (FVC) (80 to 120\%), normal forced expiratory volume for 1 st second $\left(\mathrm{FEV}_{1}\right)$ and $\mathrm{FEV}_{1} /$ FVC ratio was calculated, post-operatively all patients showed deceased FVC to levels 70 to $80 \%$, decreased $\mathrm{FEV}_{1}$ and slight increase in $\mathrm{FEV}_{1} / \mathrm{FVC}$ ratio concerning each patient. There was at first a complaint of slight difficulty of breathing which disappeared within one month.

Complications following were not major; they included 2 patients showing areas of skin necrosis at the central portion of the lower abdominal flap. Patients underwent debridement for necrotic skin and wounds were closed by secondary sutures.

During follow-up, waist measurements for all patients showed a significant decrease ranging from 5 to $16 \mathrm{~cm}$, and patients' satisfaction was very high as all patients expressed their happiness toward their figure improvement and new clothing fit Figs. (5-7).

\section{DISCUSSION}

Liposculpture, skin excision fashion and tension fascial suspensions are all appropriate means for having satisfactory results in abdominoplasty. Never the less, muscloapneurotic rehabilitation in patients with severe rectal diastasis is believed to hugely improve the aesthetic and functional outcome and give stability to the results gained, the longevity of the results is the milestone of patient satisfaction [12]. 

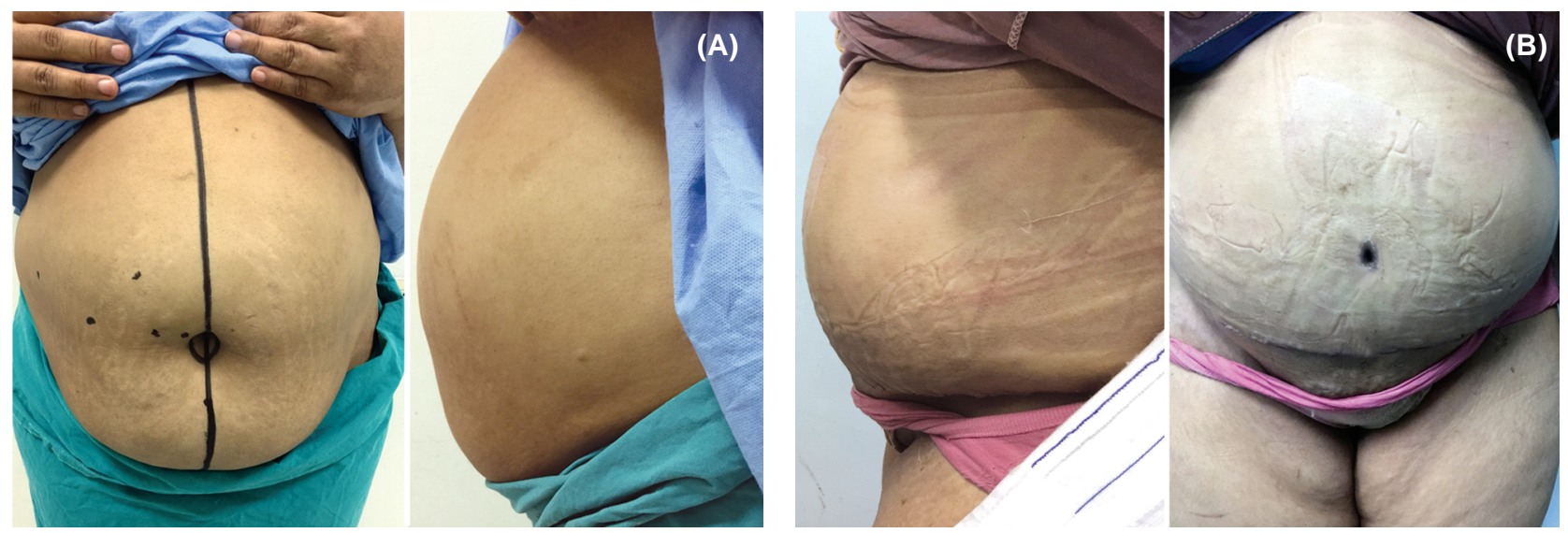

Fig. (5): Abdominoplasty patient (weight $107 \mathrm{~kg}$; height $165 \mathrm{~cm}$ ) with severe abdominal wall laxity (A) Pre-operative view anterio-posterior and lateral, (B) Post-operative view anterio-posterior and lateral.
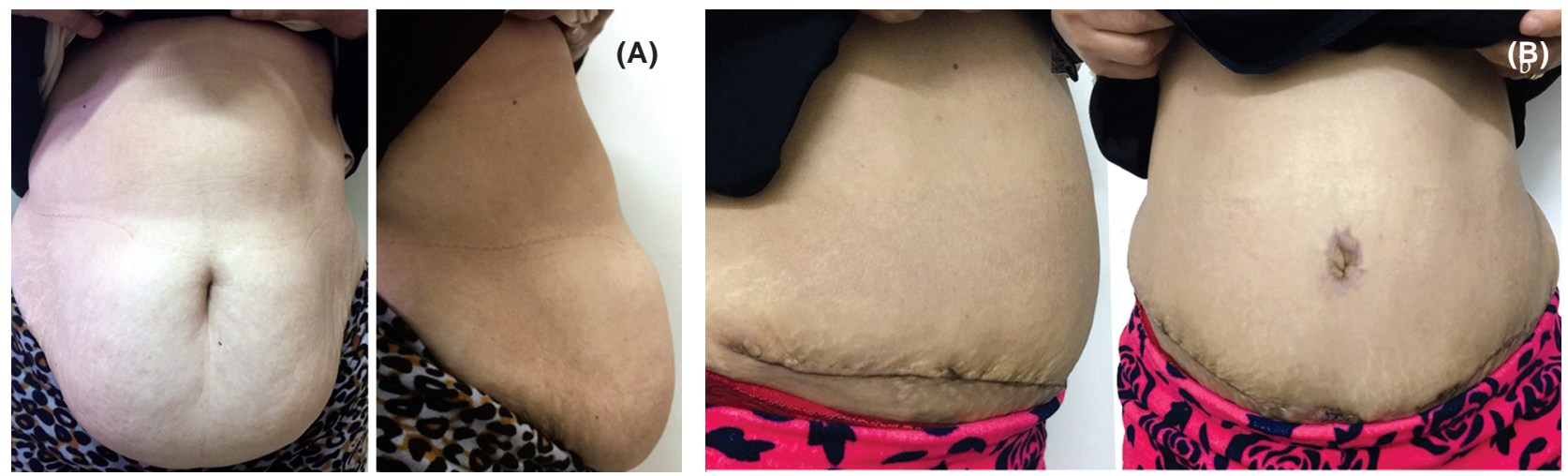

Fig. (6): Abdominoplasty patient (weight $96 \mathrm{~kg}$; height $161 \mathrm{~cm}$ ) with moderate to severe abdominal wall laxity (A) Pre-operative view anterio-posterior and lateral, (B) Post-operative view anterio-posterior and lateral.
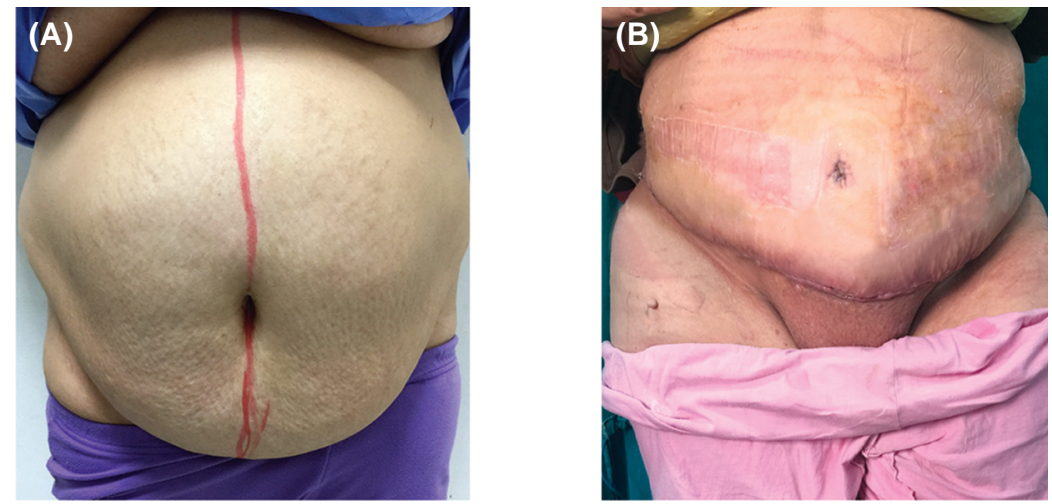

Fig. (7): Abdominoplasty patient (weight $110 \mathrm{~kg}$; height $163 \mathrm{~cm}$ ) with severe abdominal wall laxity (A) Pre-operative anterio-posterior view, (B) Post-operative anterio-posterior view: This patient showed a decrease in waist circumference $6.6 \mathrm{~cm}$.

Suture plication of the muscloapneurotic layer is commonly used in abdominoplastiy operations, but many disadvantages were reported: It doesn't treat the rectus sheath redundancy as sutures are taken in the outer tendentious sheath leaving the rectus muscle inside a wide roomy intra-rectal sheath spaces, thus limiting the function of the muscle [13]. Also it doesn't help in management of co-existing ventral hernias and can even worsen the condition by causing intestinal injury when applying the plicating sutures, in addition to stim- ulation of other weak points which will produce a clinically obvious hernia [14].

The incidence of recurrence has been attributed to suture plication [15], also orientation of rectus muscles doesn't change by suture plication causing the muscle action to separate the plication and increase incidence of recurrence [1].

Using braided sutures in multiple layers to benefit from its higher holding capacity and increase fibrosis between the recti sheath layers gives 
more stable and durable results. So with application of myofascial repair, there is a chance for creating fascial raw areas in the rectus sheath layers; promoting more collagen deposition which will cause solid healing [4-7].

Myofascial repair in addition will provide approximation and reorientation of both recti muscles inwards and medially, this will help to avoid significant centrifugal forces acting on the repair, provide effective traction on the external oblique muscles decreasing the size of waistline, and recreating a more physiological linea alba to give satisfactory aesthetic and functional outcomes [16].

The procedure performed in this study gave results similar to results described by Rameriz [1] and Nahas [16], their procedure removed excess abdominal skin, improved the waistline and tightened the lax abdominal wall musculature giving great aesthetic and functional outcomes. Their procedure gave also special attention to the importance of myofascial repair in having these great long term outcomes.

The technique that achieves the highest tension is the one that gives the best aesthetic abdominoplasty outcome [3]. However, in patients with severe abdominal laxity, the distance between both recti is very wide that even with the three layered myofascial repair; the tension is highly increased and sutures may be ripped off with increased intraabdominal pressure during coughing and constipation. The outermost layer shows higher tension than other layers and is the most affected layer during increased intra-abdominal pressure.

Rameriz [1] did not give an obvious solution for this problem. On the contrary, this study focused especially on this serious problem suggesting to excise small parts of the linea alba in order to approximate both recti and convert the redundant linea alba to a fourth layer with partial raw areas contributing to the strength of this layer when healing by fibrosis. All this will have a significant effect on decreasing the tension of sutures on myofacial repair layers especially the outermost layer, which will decrease incidence of failure of mayofacial repair and guarantee more long term stable results.

\section{Conclusion:}

Myofascial repair modification of the abdominal muscluoapnrurotic system described in this study will provide more functional and aesthetic outcomes in abdominoplasty in patients with severe abdominal laxity and give better long term stable results.
Also myofascial release and repair technique is superior to suture plication in decreasing the waist measurement and giving a better figure with less functional drop backs and less incidence of recurrence.

\section{REFERENCES}

1- Oscar M. Ramirez: Abdominoplasty and abdominal wallrehabilitation: A Comprehensive Approach. Plast. Reconstr. Surg., 105 (1): 425-35, 2000.

2- Matarasso A.: Liposuction as an adjunct to a full abdominoplasty, Plast. Reconstr. Surg., 95: 829, 1995.

3- Baroudi R. and Ferreira C.A.: Contouring the hip and the abdomen. Clin. Plast. Surg., 23: 551, 1996.

4- Pitanguy I.: Abdominoplastias. Hospital, 71 (6): 1541e56, 1967.

5- Birdsell D.C., Gavelin G.E. and Kemsley G.M..: "Stayingpower"-absorbable vs. nonabsorbable. Plast. Reconstr. Surg., 68 (5): 742-5, 1981.

6- Nahas F.X., Augusto S.M. and Ghelfond C.: Nylon versus polydioxanone in the correction of rectus diastasis. Plast. Reconstr. Surg., 107 (3): 700-6, 2001.

7- Sozer S.O. and Agullo F.J.: Triple plication in miniabdominoplasty. Aesthetic Plast. Surg., 30 (3): 263-8, 2006.

8- Van Uchelen J.H., Kon M. and Werker P.M.: The longterm dura-bility of plication of the anterior rectus sheath assessed by ultrasonography. Plast. Reconstr. Surg., 107: 1578-84, 2001.

9- Seymour N.E. and Bell R.L.: Abdominal wall, omentum, mesentery, and retroperitoneum. In: Schwartz S.I., Brunicardi F.C., eds. Schwartz's Principles of Surgery. New York: McGraw-Hill: 1267-128, 2010.

10- Nahas F.X., Ishida J., Gemperli R. and Ferreira M.C.: Abdominal-wall closure after selective aponeurotic incision and undermining. Ann. Plast. Surg., 41: 606, 1998.

11- DiBello J.N. Jr. and Moore J.H. Jr.: Sliding myofascial flap of the rectus abdominus muscles for the closure of recurrent ventral hernias. Plast. Reconstr. Surg., 98: 4649, 1996.

12- Psillakis J.M.: Plastic surgery of the abdomen with improvement in the body contour; physiopathology and treatment of the aponeurotic musculature. Clin. Plast. Surg., 11: 465, 1984

13- Hensel J.M., Lehman J.A. Jr. and Tantri M.P.: An outcomes analysis and satisfaction survey of 199 consecutive abdominoplasties. Ann. Plast. Surg., 46 (4): 357-63, 2001.

14- Garrido D.E., Aponte Y.M., Behnam A.B., Keeshin T., Sinha V., Evans K.K. and Salgado C.J.: Updates in Abdominal Wall Reconstruction. Anaplastology, 2: 1-5, 2013.

15- Cheesborough J.E. and Dumanian G.A.: Simultaneous prosthetic mesh abdominal wall reconstruction with abdominoplasty for ventral hernia and severe rectus diastasis repairs. Plast. Reconstr. Surg., 135: 268, 2015.

16- Nahas F.X. and Ferreira L.M.: Concepts on Correction of the Musculoaponeurotic Layer in Abdominoplasty. Clin. Plastic Surg., 37: 527-38, 2010. 